\title{
QSAR studies on imidazoles and sulfonamides as antidiabetic agents
}

\author{
Veerasamy RAVICHANDRAN ${ }^{* 1}$ and Rajak HARISH ${ }^{2}$ \\ ${ }^{1}$ Faculty of Pharmacy, AIMST University, Semeling-08100, Kedah, Malaysia \\ ${ }^{2}$ SLT Institute of Pharmaceutical Sciences, Guru Ghasidas University, Bilaspur, India
}

\begin{abstract}
The main objective of the present study was to establish significant and validated QSAR models for imidazoles and sulfonamides to explore the relationship between their physicochemical properties and antidiabetic activity. Two dimensional QSAR models had been developed by multiple linear regression and partial least square analysis methods, and then validated for internal and external predictions. The established 2D QSAR models were statistically significant and highly predictive. The validation methods provided significant statistical parameters with $\mathrm{q}^{2}>0.5$ and pred_r $\mathrm{r}^{2}>0.6$, which proved the predictive power of the models. The developed 2D QSAR models revealed the significance of SlogP and T_N_O_5, and Mol.Wt and SsBrE-index properties of imidazoles and sulfonamides on their antidiabetic activity, respectively. These results should prove to be an essential guide for the further design and development of new imidazoles and sulfonamides having better antidiabetic activity.
\end{abstract}

Keywords: antidiabetic, physicochemical properties, QSAR, validation.

\section{Introduction}

The discovery and development of a new chemical entity with demonstrated utility in ameliorating or curing disease is a long and arduous process. Industry statistics suggests that up to several thousand compounds are synthesized and tested; up to 100 compounds are assessed for safety; and up to 10 compounds are tested clinically in humans for every drug that is approved for medical use. Trial and error screening used to consider as the normal procedure, is becoming very costly and at the same time less efficient. Therefore, only molecules with good chance of activity should be prepared and tested. In this context, proper designing is required before synthesis of the drugs.

Diabetes is a complex and costly disease that can affect nearly every organ in the body and result in devastating consequences [1]. Diabetes is the major causes of renal failure, blindness [2], stroke, cardiovascular disease, premature and perinatal mortality [3]. Insulin is the choice of treatment for type 1 diabetes. Type 2 diabetes can be managed with a combination of different oral and injectable antidiabetic agents [4].

In rational drug design, numerous 2D QSAR studies [5-10], 3D QSAR studies [11, 12] and binding studies [13] have been reported for different group of chemical derivatives and their antidiabetic activity. With the aim of developing good antidiabetic drugs, we have selected some imidazole and sulfonamide antidiabetic compounds [14-17] to understand structural insight, which is responsible for selectivity of these derivatives towards diabetes by using QSAR analysis. The series of compounds had shown well defined activity. There was high structural diversity and a sufficient range of the biological activity in the series of compounds selected for the present study. The developed QSAR models were statistically significant and could efficiently guide to design imidazole and sulfonamide derivatives with better antidiabetic potential.

\section{Experimental}

\subsection{Software}

CS Chem Office 2004 (Cambridge Soft Corp., Cambridge, USA, http://www. cambridgesoft.com) and the molecular modeling studies were carried out in Vlife MDS 4.3 (VLife Sci Tech Private Ltd, India, WwW.vlifesciences.com).

\subsection{Sketching of molecules}

The .mol files of structures (Imidazole and sulfonamide antidiabetic compounds [14-17]), which were drawn and cleaned up in Chem 3D, were transferred to VLife MDS.

\subsection{Energy minimization}

"The geometry of the 3D structures was optimized to local minima by Merck Molecular Force Field (MMFF) by considering distance-dependent dielectric constant of 1.0 , convergence criterion or root-mean-square (RMS) gradient at $0.001 \mathrm{kcal} / \mathrm{mol} \AA$ and the iteration limit to 10,000 . Most stable structure for each compound was generated and saved as .mol 2 files for computing various physico-chemical and alignment independent descriptors" [18].

\subsection{Calculation of descriptor (Independent variable)}

The energy-minimized structures were used for the calculation of the various 2D descriptors like physicochemical (200 in numbers) and other alignment independent descriptors (700 in numbers). The preprocessing of the independent variables (i.e., 2D descriptors) was done by removing invariables and variable exclusion was done for constant variable or near constant variable at paired correlation. Finally all

\footnotetext{
* Corresponding author.E-mail address: phravi75@rediffmail.com, sameshyaravi@gmail.com (Veerasamy Ravichandran)
} 
together 300 descriptors were selected to develop models.

\subsection{Training and test set selection}

Division of compounds into training and test data set was done by using sphere exclusion (SE) and random selection methods. The dissimilarity value 1 and 1.5 was used in sphere exclusion method, where the dissimilarity value is the sphere exclusion radius. In random selection method selection of training set compounds with four trials 70, 75, 80 and $85 \%$ were tried [19]. The reported $\mathrm{IC}_{50}$ or $\mathrm{Ki}$ values of the selected series of compounds for the present QSAR study as their negative logarithmic concentrations $\left[-\log \mathrm{IC}_{50}\right.$ or $\mathrm{pIC}_{50}$ or $\left.\mathrm{pKi}\right]$, where $\mathrm{IC}_{50}$ is the micro molar concentration of the compounds producing $50 \%$ inhibition in the glucagon receptor activity [14] and $\mathrm{Ki}$ is carbonic anhydrase II inhibitor activity [15-17].

\subsection{Feature selection and model development}

In the present study, stepwise (SW) forward-backward variable selection [20], genetic algorithms (GA) [21] and simulated annealing (SA) [22] based feature selection procedures were used to build QSAR models. The crosscorrelation limit 0.5 , the number of variables 5 , and the term selection criteria $\mathrm{q}^{2}$ was used to build QSAR models. The variance cutoff of 0 and auto-scaling with number of random iterations 100 was used to normalize the independent variables.

The stepping criteria for inclusion of predictor variable $\mathrm{F}=4$ and exclusion of predictor variable $\mathrm{F}=$ 3.99 was used in SW forward-backward variable selection algorithm. Population 10, number of generations 1000 and speed of 9999 was used in GA method. The maximum and minimum temperature used in SA method was $100 \mathrm{~K}$ and $0.01 \mathrm{~K}$, and it was decreased by 5 units with 100 iterations at that particular temperature [18].

Multiple linear regression and partial least square analysis were used to find the relation between the dependent and independent variables. The relationship between dependent variable and various independent variables were established by using QSAR module of VLife MDS software.

\subsection{Model quality and validation}

The developed QSAR models are evaluated using the following statistical measures: $\mathrm{n}$ (the number of compounds in regression); $\mathrm{k}$ (number of variables); $\mathrm{DF}$ (degree of freedom); $\mathrm{r}^{2}$ (squared correlation coefficient); $\mathrm{r}^{2} \mathrm{se}$ (standard error of $\mathrm{r}^{2}$ ); $\mathrm{F}$ test (Fischer's value); $\mathrm{q}^{2}$ (cross-validated squared correlation coefficient); $\mathrm{q}^{2} \_\mathrm{se}$ (standard error of $\mathrm{q}^{2}$ ); pred_ $\mathrm{r}^{2}\left(\mathrm{r}^{2}\right.$ for external test set); pred_r ${ }^{2}$ se (standard error of pred_r ${ }^{2}$ ); $\mathrm{Z}$ score $(\mathrm{Z}$ score calculated by the randomization test); best_ran_q ${ }^{2}$ (highest $\mathrm{q}^{2}$ value in the randomization test); best_ran_ $\mathrm{r}^{2}$ (highest $\mathrm{r}^{2}$ value in the randomization test). A QSAR model is considered to be predictive, if the following conditions are satisfied: $\mathrm{r}^{2}>0.7, \mathrm{q}^{2}>0.5$ and pred_ $\mathrm{r}^{2}>$ 0.6 [23]. The quality and validation parameters of QSAR models are compiled and discussed in detail somewhere by Ravichandran et al. [24].

\section{Results and discussion}

\section{Series I: Triaryl imidazoles}

The glucagon antagonist activity and structure of 27 compounds (Table 1) was taken from the study reported by Chang et al. after excluding 11 molecules which were not having a well-defined biological activity, and 3 molecules that are not congeners to the rest of the dataset [14]. Different feature selection and model development methods were used to develop 2D QSAR models. One of the best developed models was Eq. (1). The criteria's used to get this model were: random training and test set selection method (70\%), stepwise forward-backward variable selection method, model development by multiple linear regression (MLR), test set compounds: $\mathbf{4}$, $5,7,10,15,16,21,25$, and outliers: compound 8 .

$$
\begin{aligned}
& \mathrm{pIC}_{50}=1.923+0.753( \pm 0.062) \mathrm{S} \log \mathrm{P}+0.591( \pm 0.201) \\
& \mathrm{T} \_\mathrm{N} \_\mathrm{O} \_5 \quad \text { Eq. }(1) \\
& \mathrm{n}=18, \mathrm{r}^{2}=0.917, \mathrm{r}^{2} \mathrm{se}=0.302, \mathrm{q}^{2}=0.880, \mathrm{q}^{2} \mathrm{se}=0.364, \\
& \mathrm{~F}_{2,15}=83.191, \mathrm{pred}^{2} \mathrm{r}^{2}=0.830, \mathrm{pred}_{-} \mathrm{r}^{2} \mathrm{se}=0.425, \mathrm{Z} \\
& \mathrm{Score} \mathrm{r}^{2}=8.681, \mathrm{Z} \text { Score } \mathrm{q}^{2}=7.563, \text { Best Rand } \mathrm{r}^{2}= \\
& 0.387, \text { Best Rand } \mathrm{q}^{2}=0.138 \\
& \text { Eq. (1) could explain 91.7\% and predict 83.0\% of the } \\
& \text { variance of the antidiabetic data. There was no inter- } \\
& \text { correlation between the descriptors. The parameters } \\
& \text { involved in the selected model (SlogP, T_N_O_5) and } \\
& \text { the calculated antidiabetic activity by Eq. (1) are given in } \\
& \text { Table } 2 \text {. }
\end{aligned}
$$

Table 1. The structures of triaryl imidazoles with their glucagons antagonist activity

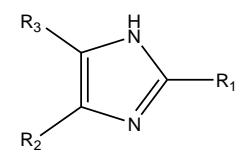

\begin{tabular}{|ccccc|}
\hline Compounds & $\mathbf{R}_{\mathbf{1}}$ & $\mathbf{R}_{\mathbf{2}}$ & $\mathbf{R}_{\mathbf{3}}$ & $\mathbf{p I C}_{\mathbf{5 0}}$ \\
\hline $\mathbf{1}$ & (4-Br)Ph & (4-F)Ph & 4-pyridyl & 6.569 \\
$\mathbf{2}$ & (3-Br)Ph & (4-F)Ph & 4-pyridyl & 5.854 \\
$\mathbf{3}$ & (4-Cl)Ph & (4-F)Ph & 4-pyridyl & 6.398 \\
$\mathbf{4}$ & (4-F)Ph & (4-F)Ph & 4-pyridyl & 5.699 \\
$\mathbf{5}$ & (4-I)Ph & (4-F)Ph & 4-pyridyl & 6.292 \\
$\mathbf{6}$ & $(4-\mathrm{Me}) \mathrm{Ph}$ & (4-F)Ph & 4-pyridyl & 5.886 \\
$\mathbf{7}$ & (4-iPr)Ph & (4-F)Ph & 4-pyridyl & 6.155 \\
$\mathbf{8}$ & (4-Ph)Ph & (4-F)Ph & 4-pyridyl & 5.000 \\
$\mathbf{9}$ & $\left(4-\mathrm{NH}_{2}\right) \mathrm{Ph}$ & (4-F)Ph & 4-pyridyl & 5.699 \\
\hline
\end{tabular}




\begin{tabular}{|c|c|c|c|c|}
\hline Compounds & $\mathbf{R}_{\mathbf{1}}$ & $\mathbf{R}_{2}$ & $\mathbf{R}_{3}$ & pIC50 \\
\hline 10 & $(4-\mathrm{OMe}) \mathrm{Ph}$ & (4-F)Ph & 4-pyridyl & 4.886 \\
\hline 11 & $(4-\mathrm{CN}) \mathrm{Ph}$ & $(4-F) P h$ & 4-pyridyl & 5.097 \\
\hline 12 & (4-COOMe)Ph & $(4-\mathrm{F}) \mathrm{Ph}$ & 4-pyridyl & 5.060 \\
\hline 13 & (4-SMe)Ph & (4-F)Ph & 4-pyridyl & 6.310 \\
\hline 14 & $(4-\mathrm{Br}) \mathrm{Ph}$ & $\mathrm{Ph}$ & 4-pyridyl & 6.107 \\
\hline 15 & (4-Cl)Ph & $(4-F) P h$ & 3-Me(4-pyridyl) & 5.959 \\
\hline 16 & (4-Cl)Ph & (4-Cl)Ph & 4-pyridyl & 6.721 \\
\hline 17 & $(4-\mathrm{Cl}) \mathrm{Ph}$ & $(4-I) P h$ & 4-pyridyl & 6.886 \\
\hline 18 & $(4-\mathrm{Cl}) \mathrm{Ph}$ & $(4-\mathrm{Ph}) \mathrm{Ph}$ & 4-pyridyl & 6.854 \\
\hline 19 & $(4-\mathrm{Cl}) \mathrm{Ph}$ & $(4-\mathrm{t}-\mathrm{Bu}) \mathrm{Ph}$ & 4-pyridyl & 6.886 \\
\hline 20 & $(4-\mathrm{Cl}) \mathrm{Ph}$ & $(4-n-B u) P h$ & 4-pyridyl & 7.131 \\
\hline 21 & (4-Cl)Ph & $(3-\mathrm{Ph}) \mathrm{Ph}$ & 4-pyridyl & 7.215 \\
\hline 22 & (4-Cl)Ph & $(2-\mathrm{OPh}) \mathrm{Ph}$ & 4-pyridyl & 8.187 \\
\hline 23 & (4-Cl)Ph & (3-OPh)Ph & 4-pyridyl & 7.886 \\
\hline 24 & (4-Cl)Ph & $(4-\mathrm{OPh}) \mathrm{Ph}$ & 4-pyridyl & 7.569 \\
\hline 25 & (4-Cl)Ph & $(2-\mathrm{O}-\mathrm{n}-\mathrm{Bu}) \mathrm{Ph}$ & 4-pyridyl & 8.071 \\
\hline 26 & $(4-\mathrm{Cl}) \mathrm{Ph}$ & $\left(2,4-(\mathrm{O}-\mathrm{n}-\mathrm{Pr})_{2}\right) \mathrm{Ph}$ & 4-pyridyl & 7.886 \\
\hline 27 & (4-Cl)Ph & $\left(2,4-(\mathrm{O}-\mathrm{n}-\mathrm{Bu})_{2}\right) \mathrm{Ph}$ & 4-pyridyl & 8.187 \\
\hline
\end{tabular}

Table 2. Descriptors involved in $2 D$ QSAR model Eq. (1) for glucagons receptor antagonistic activity of triaryl imidazoles and their predicted activity

\begin{tabular}{|ccccc|}
\hline Compounds & SlogP & T_N_O_5 & Actual activity (pIC50) & $\begin{array}{c}\text { Predicted activity } \\
\text { (pIC50) }\end{array}$ \\
\hline $\mathbf{1}$ & 5.707 & 0 & 6.569 & 6.217 \\
$\mathbf{2}$ & 5.707 & 0 & 5.854 & 6.217 \\
$\mathbf{3}$ & 5.598 & 0 & 6.398 & 6.135 \\
$\mathbf{4}^{\text {a }}$ & 5.084 & 0 & 5.699 & 5.749 \\
$\mathbf{5}^{\mathbf{a}}$ & 5.549 & 0 & 6.292 & 6.099 \\
$\mathbf{6}$ & 5.253 & 0 & 5.886 & 5.876 \\
$\mathbf{7}^{\mathbf{a}}$ & 6.068 & 0 & 6.155 & 6.489 \\
$\mathbf{8}$ & 6.612 & 0 & $*$ & $*$ \\
$\mathbf{9}$ & 4.527 & 0 & 5.699 & 5.330 \\
$\mathbf{1 0}$ & 4.953 & 0 & 4.886 & 5.650 \\
$\mathbf{1 1}$ & 4.816 & 0 & 5.097 & 5.547 \\
$\mathbf{1 2}$ & 4.731 & 0 & 5.06 & 5.483 \\
$\mathbf{1 3}$ & 5.667 & 0 & 6.31 & 6.187 \\
$\mathbf{1 4}$ & 5.568 & 0 & 6.107 & 6.113 \\
$\mathbf{1 5}$ & 5.907 & 0 & 5.959 & 6.368 \\
$\mathbf{1 6}$ & 6.112 & 0 & 6.721 & 6.522 \\
$\mathbf{1 7}$ & 6.064 & 0 & 6.886 & 6.486 \\
$\mathbf{1 8}$ & 7.126 & 0 & 6.854 & 7.285 \\
$\mathbf{1 9}$ & 6.757 & 0 & 6.886 & 7.008 \\
$\mathbf{2 0}$ & 6.802 & 0 & 7.131 & 7.041 \\
$\mathbf{2 1}$ & 7.126 & 0 & 7.215 & 7.285 \\
$\mathbf{2 2}$ & 7.251 & 1 & 8.187 & 7.970 \\
$\mathbf{2 3}$ & 7.251 & 1 & 7.886 & 7.970 \\
$\mathbf{2 4}$ & 7.251 & 0 & 7.569 & 7.379 \\
$\mathbf{2 5}$ & 6.638 & 1 & 8.071 & 7.509 \\
$\mathbf{2 6}$ & 7.037 & 1 & 7.886 & 8.809 \\
$\mathbf{2 7}$ & 7.817 & 1 & 8.187 & \\
\hline
\end{tabular}

a - indicates test set compounds, * - indicates outliers

The correlation of experimental activity against predicted activities by Eq. (1) is graphically represented in Figure 1. The closeness of the actual and predicted activity by Eq. (1) for training and test set compounds are shown in Figure 2 and 3, respectively.

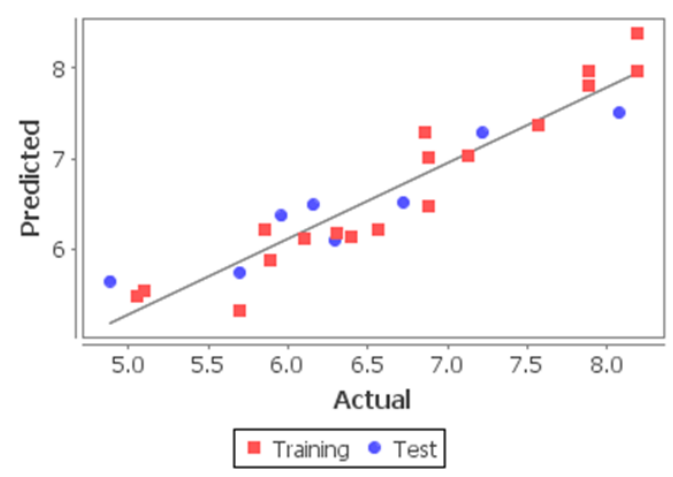

Figure 1. Fitness plot between the experimental and predicted activities by Eq. (1) 


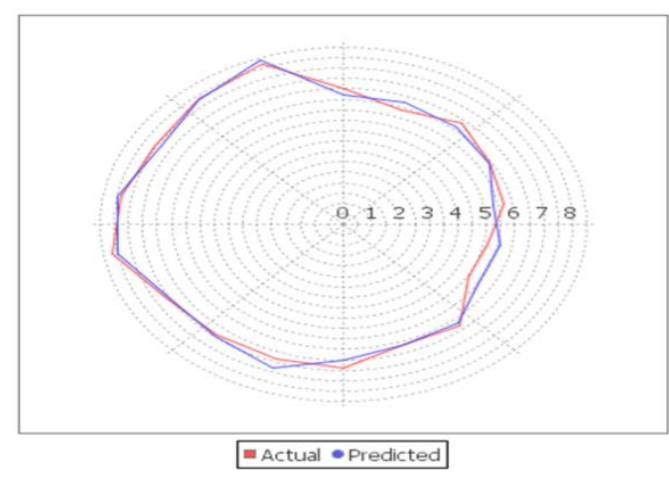

Figure 2. Radar plot depicting closeness between the actual and predicted activity of training set compounds by Eq. (1)

The selected model was good in internal prediction $\left(\mathrm{q}^{2}=\right.$ 0.880 ) and external prediction $\left(\right.$ pred_ $\left.\mathrm{r}^{2}=0.830\right)$. The good results in our original model are not due to a chance correlation or structural dependency of the compounds was buttressed by the low randomized $r^{2}(0.287)$ and $q^{2}$ $(0.138)$ values.

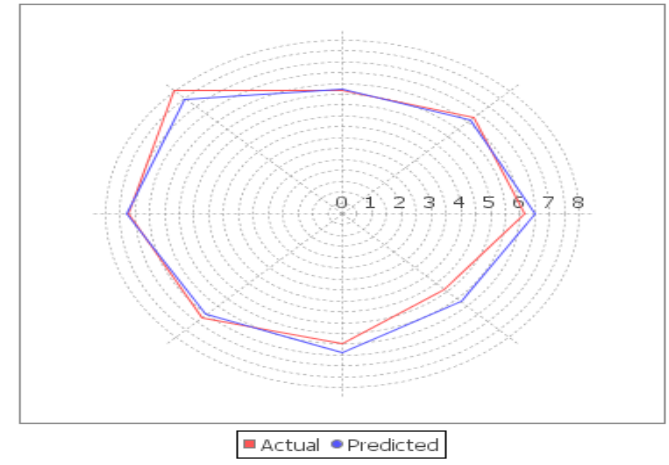

Figure 3. Radar plot depicting closeness between the actual and predicted activity of test set compounds by Eq. (1)

The developed MLR model reveals that the descriptor T_N_O_5 which is an alignment independent descriptor showed positive contribution. Such positive effect indicates that the antidiabetic activity is increased with increase in the count of number of nitrogen atom (single, double or triple bonded) separated from any oxygen atom (single, double or triple bonded) by 5 bond distance in a molecule. These findings were supported by the compounds 22, 23, 24, 25, 26 and 27 which have substitution of 2-OPh- $\mathrm{Ph}, 3-\mathrm{OPh}-\mathrm{Ph}, 4-\mathrm{OPh}-\mathrm{Ph}$, 2-O$n B u-P h, 2,4-(\mathrm{O}-n \mathrm{Pr})_{2}-\mathrm{Ph}$ or $2,4-(\mathrm{O}-n \mathrm{Bu})_{2}-\mathrm{Ph}$ at $\mathrm{C}-4$ position $\left(\mathrm{R}_{2}\right)$ of imidazole. The other descriptor SlogP signifies $\log$ of the octanol/water partition coefficient (Including implicit hydrogen). The positive contribution SlogP suggests that the antidiabetic activity is increased with substitution of strong hydrophobic groups. These findings were supported by the compounds 18 to 27 which have substitution of strong hydrophobic groups specifically at C-4 position of imidazole $\left(\mathrm{R}_{2}\right)$. Modification of the parameters T_N_O_5 and SlogP for the present series of compounds will lead to good effect on antidiabetic activity.

\section{Series II: Aromatic sulfonamides}

The carbonic anhydrase II inhibitory activity and structure of 47 compounds (Table 3) were used for the present study [15-17]. Different feature selection and model development methods were used to develop 2D QSAR models. One of the best developed models was Eq. (2). The criteria's used to get this model were: random training and test set selection method (80\%), stepwise forward-backward variable selection method, model development by multiple linear regression (MLR), and test set compounds: 2, 12, 15, 19, 21, 23, 25, 28, 36, 45.

$$
\begin{aligned}
& \text { pKi }=-0.605+0.001( \pm 0.000) \text { Mol.Wt. }+0.2446 \\
& ( \pm 0.126) \text { SsBrE-index } \quad \text { Eq. }(2)
\end{aligned}
$$

$\mathrm{n}=37, \mathrm{r}^{2}=0.663, \mathrm{r}^{2} \mathrm{se}=0.085, \mathrm{q}^{2}=0.623, \mathrm{q}^{2} \mathrm{se}=0.089$, $\mathrm{F}_{2,34}=33.375$, pred_r $\mathrm{r}^{2}=0.714$, pred_r ${ }^{2} \mathrm{se}=0.079, \mathrm{Z}$ Score $\mathrm{r}^{2}=13.021, \mathrm{Z}$ Score $\mathrm{q}^{2}=5.647$, Best Rand $\mathrm{r}^{2}=0.201$, Best Rand $\mathrm{q}^{2}=0.082$

Table 3. The structures of para-substituted aromatic sulfonamides with their carbonic anhydrase II inhibitory activity<smiles>NS(=O)(=O)c1ccc(C(=O)O)cc1</smiles><smiles>NNC(=O)c1ccc(S(N)(=O)=O)cc1</smiles><smiles>NNC(=O)NNC(=O)c1ccc(S(N)(=O)=O)cc1</smiles>

1

\begin{tabular}{|l|}
\hline Com \\
\hline \\
\hline
\end{tabular}

2

3-9

$\mathbf{p K}_{\mathbf{i}}$

\begin{tabular}{lcc|}
$\mathbf{1}$ & $\mathbf{R}$ & $\mathbf{p K}$ \\
$\mathbf{2}$ & - & -0.382 \\
$\mathbf{3}$ & - & -0.321 \\
$\mathbf{4}$ & $3,4-\mathrm{Cl}_{2} \mathrm{C}_{6} \mathrm{H}_{3}$ & -0.047 \\
$\mathbf{5}$ & $4-\mathrm{Ac}-\mathrm{C}_{6} \mathrm{H}_{4}$ & -0.070 \\
$\mathbf{6}$ & $4-\mathrm{EtOOC}-\mathrm{C}_{6} \mathrm{H}_{4}$ & 0.020 \\
$\mathbf{7}$ & $4-\mathrm{Br}_{6}-\mathrm{C}_{6} \mathrm{H}_{4}$ & 0.064 \\
$\mathbf{8}$ & $4-\mathrm{Ph}_{6}-\mathrm{C}_{6} \mathrm{H}_{4}$ & -0.018 \\
$\mathbf{9}$ & $4-\mathrm{PhO}_{4}-\mathrm{C}_{6} \mathrm{H}_{4}$ & -0.099 \\
& $4-\mathrm{PhCH}_{2}-\mathrm{C}_{6} \mathrm{H}_{4}$ & -0.070
\end{tabular}




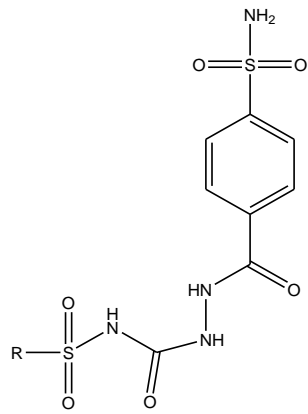

10-14

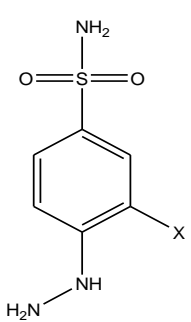

15-16

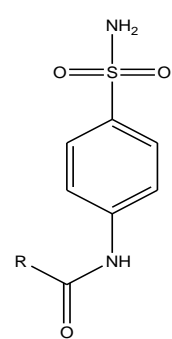

17-26

\begin{tabular}{|ccc|}
\hline Compounds & $\mathbf{R}$ & $\mathbf{p \mathbf { K } _ { \mathbf { i } }}$ \\
\hline $\mathbf{1 0}$ & $\mathrm{Ph}$ & -0.102 \\
$\mathbf{1 1}$ & $2-\mathrm{Me}-\mathrm{C}_{6} \mathrm{H}_{4}$ & -0.084 \\
$\mathbf{1 2}$ & $4-\mathrm{Me}_{6} \mathrm{C}_{6} \mathrm{H}_{4}$ & -0.084 \\
$\mathbf{1 3}$ & $4-\mathrm{F}-\mathrm{C}_{6} \mathrm{H}_{4}$ & -0.079 \\
$\mathbf{1 4}$ & $4-\mathrm{Cl}_{6} \mathrm{C}_{4}$ & -0.059 \\
$\mathbf{1 5}$ & $\mathrm{X}=\mathrm{F}$ & -0.346 \\
$\mathbf{1 6}$ & $\mathrm{X}=\mathrm{Cl}$ & -0.325 \\
$\mathbf{1 7}$ & $\mathrm{Me}$ & -0.334 \\
$\mathbf{1 8}$ & $\mathrm{CF}_{3}$ & -0.266 \\
$\mathbf{1 9}$ & $\mathrm{Et}$ & -0.317 \\
$\mathbf{2 0}$ & $n-\mathrm{Pr}$ & -0.281 \\
$\mathbf{2 1}$ & $i-\mathrm{Pr}$ & -0.299 \\
$\mathbf{2 2}$ & $n-\mathrm{Bu}$ & -0.264 \\
$\mathbf{2 3}$ & $t-\mathrm{Bu}_{5}$ & -0.264 \\
$\mathbf{2 4}$ & $n-\mathrm{C}_{5} \mathrm{H}_{11}$ & -0.264 \\
$\mathbf{2 5}$ & $\mathrm{Ph}$ & -0.264 \\
$\mathbf{2 6}$ & $\mathrm{C}_{6} \mathrm{~F}_{5}$ & -0.143 \\
\hline
\end{tabular}
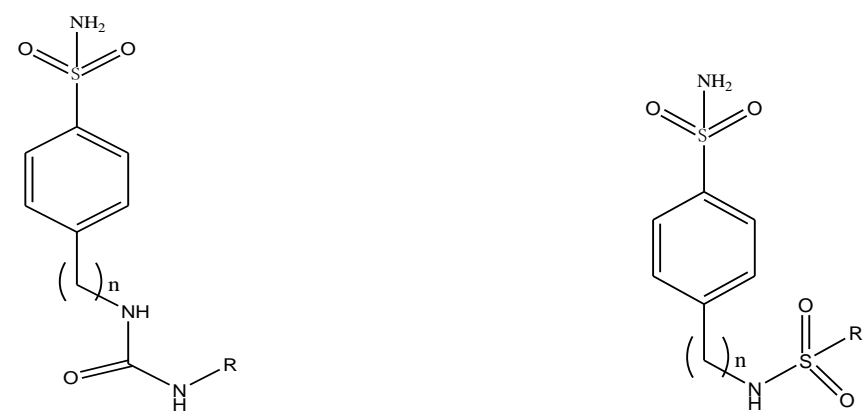

27-30

31-35

\begin{tabular}{|cccc|}
\hline Compounds & $\mathbf{n}$ & $\mathbf{R}$ & pKi \\
\hline $\mathbf{2 7}$ & 0 & $\mathrm{Ph}$ & -0.237 \\
$\mathbf{2 8}$ & 1 & $\mathrm{Ph}$ & -0.219 \\
$\mathbf{2 9}$ & 2 & $\mathrm{Ph}$ & -0.202 \\
$\mathbf{3 0}$ & 2 & $3,4-\mathrm{Cl}_{2} \mathrm{C}_{6} \mathrm{H}_{3}$ & -0.115 \\
$\mathbf{3 1}$ & $\mathrm{Ph}$ & -0.211 \\
$\mathbf{3 2}$ & 0 & $\mathrm{Ph}$ & -0.193 \\
$\mathbf{3 3}$ & 1 & $\mathrm{Ph}$ & -0.175 \\
$\mathbf{3 4}$ & 2 & $4-\mathrm{FC}_{6} \mathrm{H}_{4}$ & -0.188 \\
$\mathbf{3 5}$ & 0 & $4-\mathrm{AcNHC}_{6} \mathrm{H}_{4}$ & -0.103 \\
\hline
\end{tabular}<smiles>Nc1ccc(S(N)(=O)=O)cc1</smiles>

36<smiles>NNc1ccc(S(N)(=O)=O)cc1</smiles>

37<smiles>NCc1ccc(S(N)(=O)=O)cc1</smiles>

38 
<smiles>NCCc1ccc(S(N)(=O)=O)cc1</smiles>

39<smiles>[X]c1cc(S(N)(=O)=O)ccc1N</smiles>

40-43

\begin{tabular}{|ccc|}
\hline Compounds & $\mathbf{X}$ & $\mathbf{p K}_{\mathbf{i}}$ \\
\hline $\mathbf{3 6}$ & - & -0.387 \\
$\mathbf{3 7}$ & - & -0.369 \\
$\mathbf{3 8}$ & - & -0.370 \\
$\mathbf{3 9}$ & - & -0.352 \\
$\mathbf{4 0}$ & $\mathrm{F}$ & -0.365 \\
$\mathbf{4 1}$ & $\mathrm{Cl}$ & -0.344 \\
$\mathbf{4 2}$ & $\mathrm{Br}$ & -0.194 \\
$\mathbf{4 3}$ & $\mathrm{I}$ & -0.229 \\
\hline
\end{tabular}<smiles>Nc1c(S(N)(=O)=O)cc(S(N)(=O)=O)c(Cl)c1Cl</smiles>

44<smiles>Nc1cc(Cl)c(S(N)(=O)=O)cc1S(N)(=O)=O</smiles>

45<smiles>NS(=O)(=O)c1ccc(CO)cc1</smiles>

46

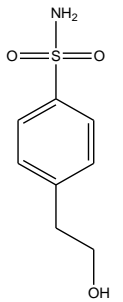

47

\begin{tabular}{|ccc|}
\hline Compounds & $\mathbf{R}$ & $\mathbf{p K}_{\mathbf{i}}$ \\
\hline $\mathbf{4 4}$ & - & -0.201 \\
$\mathbf{4 5}$ & - & -0.244 \\
$\mathbf{4 6}$ & - & -0.369 \\
$\mathbf{4 7}$ & - & -0.351 \\
\hline
\end{tabular}

Table 4. Descriptors involved in 2D QSAR model Eq. (2) for para-substituted aromatic sulfonamides as carbonic anhydrase II inhibitors with their actual and predicted activities

\begin{tabular}{|ccccc|}
\hline Compounds & Molecular weight & $\begin{array}{c}\text { SsBrE- } \\
\text { index }\end{array}$ & $\begin{array}{c}\text { Actual activity } \\
\left(\mathbf{p K}_{\mathbf{i}}\right)\end{array}$ & $\begin{array}{c}\text { Predicted activity } \\
(\mathbf{p K})\end{array}$ \\
\hline $\mathbf{1}$ & 201.203 & 0 & -0.382 & -0.382 \\
$\mathbf{2}^{\mathbf{a}}$ & 215.233 & 0 & -0.321 & -0.321 \\
$\mathbf{3}$ & 403.245 & 0 & -0.047 & -0.047 \\
$\mathbf{4}$ & 376.393 & 0 & -0.070 & -0.07 \\
$\mathbf{5}$ & 408.435 & 0 & 0.020 & 0.02 \\
$\mathbf{6}$ & 413.252 & 0.573 & 0.064 & 0.064 \\
$\mathbf{7}$ & 410.453 & 0 & -0.018 & -0.018 \\
$\mathbf{8}$ & 426.453 & 0 & -0.099 & -0.099 \\
$\mathbf{9}$ & 426.496 & 0 & -0.070 & -0.07 \\
$\mathbf{1 0}$ & 398.421 & 0 & -0.102 & -0.262 \\
$\mathbf{1 1}$ & 412.447 & 0 & -0.084 & -0.239 \\
$\mathbf{1 2}$ & 412.447 & 0 & -0.084 & 0.004 \\
$\mathbf{1 3}$ & 416.411 & 0 & -0.079 & 0.01 \\
$\mathbf{1 4}$ & 432.865 & 0 & -0.059 & 0.018 \\
$\mathbf{1 5}$ & 205.213 & 0 & -0.346 & -0.232 \\
$\mathbf{1 6}$ & 221.667 & 0 & -0.325 & -0.274 \\
$\mathbf{1 7}$ & 214.245 & 0 & -0.334 & -0.379 \\
$\mathbf{1 8}$ & 268.216 & 0 & -0.266 & -0.327 \\
$\mathbf{1 9}$ & 228.272 & 0 & -0.317 & -0.374 \\
$\mathbf{2 0}$ & 256.326 & 0 & -0.281 & -0.372 \\
$\mathbf{2 1}$ & 242.299 & 0 & -0.299 & -0.382 \\
$\mathbf{2 2}$ & 270.353 & 0 & -0.264 & -0.367 \\
\hline
\end{tabular}




\begin{tabular}{|ccccc|}
\hline Compounds & Molecular weight & $\begin{array}{c}\text { SsBrE- } \\
\text { index }\end{array}$ & $\begin{array}{c}\text { Actual activity } \\
\left(\mathbf{p K}_{\mathbf{i}}\right)\end{array}$ & $\begin{array}{c}\text { Predicted activity } \\
\left(\mathbf{p K}_{\mathbf{i}}\right)\end{array}$ \\
\hline $\mathbf{2 3}^{\mathbf{a}}$ & 270.353 & 0 & -0.264 & -0.373 \\
$\mathbf{2 4}$ & 270.353 & 0 & -0.264 & -0.255 \\
$\mathbf{2 5}^{\mathbf{a}}$ & 270.353 & 0 & -0.264 & -0.195 \\
$\mathbf{2 6}$ & 366.268 & 0 & -0.143 & -0.09 \\
$\mathbf{2 7}$ & 291.331 & 0 & -0.237 & -0.377 \\
$\mathbf{2 8}^{\mathbf{a}}$ & 305.358 & 0 & -0.219 & -0.306 \\
$\mathbf{2 9}$ & 319.384 & 0 & -0.202 & -0.273 \\
$\mathbf{3 0}$ & 388.274 & 0 & -0.115 & -0.047 \\
$\mathbf{3 1}$ & 312.37 & 0 & -0.211 & -0.228 \\
$\mathbf{3 2}$ & 326.397 & 0 & -0.193 & -0.205 \\
$\mathbf{3 3}$ & 340.424 & 0 & -0.175 & -0.161 \\
$\mathbf{3 4}$ & 330.361 & 0 & -0.188 & 0.02 \\
$\mathbf{3 5}$ & 397.476 & 0 & -0.103 & -0.273 \\
$\mathbf{3 6}$ & 172.208 & 0 & -0.387 & -0.394 \\
$\mathbf{3 7}$ & 187.222 & 0 & -0.369 & -0.399 \\
$\mathbf{3 8}$ & 186.235 & 0 & -0.370 & -0.348 \\
$\mathbf{3 9}$ & 200.262 & 0 & -0.352 & -0.343 \\
$\mathbf{4 0}$ & 190.198 & 0 & -0.365 & -0.25 \\
$\mathbf{4 1}$ & 206.653 & 0 & -0.344 & -0.31 \\
$\mathbf{4 2}$ & 251.104 & 0.383 & -0.194 & -0.205 \\
$\mathbf{4 3}$ & 298.108 & 0 & -0.229 & -0.266 \\
$\mathbf{4 4}$ & 320.177 & 0 & -0.201 & -0.161 \\
$\mathbf{4 5}$ & 285.732 & 0 & -0.244 & -0.273 \\
$\mathbf{4 6}$ & 187.219 & 0 & -0.369 & -0.322 \\
$\mathbf{4 7}$ & 201.246 & 0 & -0.351 & -0.31 \\
\hline
\end{tabular}

a - indicates test set compound

Eq. (2) could explain $66.3 \%$ and predict $71.4 \%$ of the variance of the carbonic anhydrase II inhibitory activity data. There was no inter-correlation between the descriptors. The parameters involved in the selected model (Molecular weight and SsBrE-index) and the calculated antidiabetic activity by Eq. (2) are given in Table 4.

The correlation of experimental activity against predicted activities by Eq. (2) is graphically represented in Figure 4. The closeness of the actual and predicted activity by Eq. (2) for training and test set compounds are shown in Figure 5 and 6 , respectively. The selected model was good in internal prediction $\left(\mathrm{q}^{2}=0.846\right)$ and external prediction (pred_r $r^{2}=0.714$ ). The good results in our original model are not due to a chance correlation or structural dependency of the compounds was buttressed by the low randomized $\mathrm{r}^{2}(0.201)$ and $\mathrm{q}^{2}(0.082)$ values.

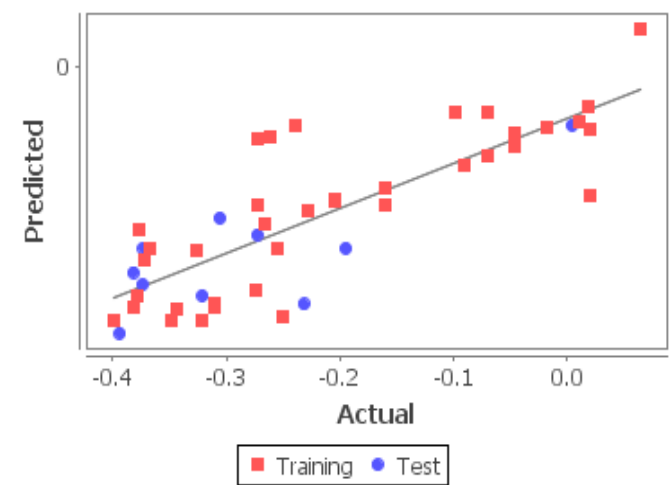

Figure 4. Fitness plot between the experimental and predicted activities by Eq. (2)

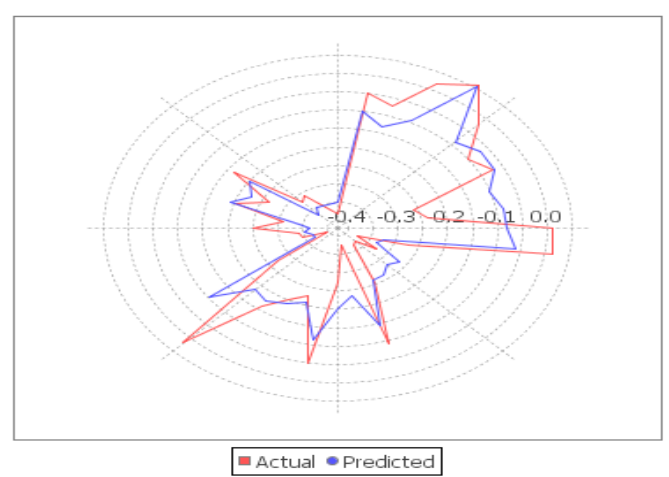

Figure 5. Radar plot depicting closeness between the actual and predicted activity of training set compounds by Eq. (2)

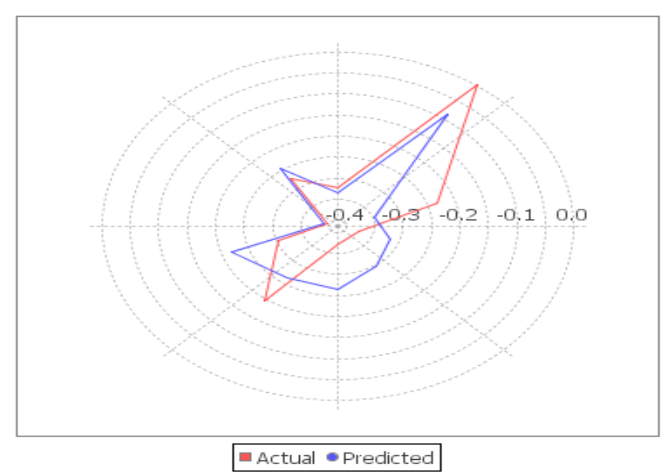

Figure 6. Radar plot depicting closeness between the actual and predicted activity of test set compounds by Eq. (2)

The developed MLR model reveals that the descriptor molecular weight showed positive contribution. Such positive effect indicates that the antidiabetic activity is increased with substitution of bulky groups in the compounds. These findings were supported by the compounds 5, 6, 12, 13 and 14 which have high molecular weight. The other descriptor, SsBrE-index is an electrotopological indices descriptor influencing 
activity variation and is directly contributing to activity. This descriptor reveals the importance of presence of number of bromine atom connected with one single bond in the compounds. Carbonic anhydrase II inhibitory activity of sulfonamides is increased with substitution of more bromine. Modification of the parameters molecular weight and SsBrE-index in the present series of sulfonamide compounds will lead to good effect on antidiabetic activity.

\section{Conclusion}

In the present study, statistically significant and highly predictive 2D QSAR models were developed for some antidiabetic compounds. The QSAR models were validated by standard statistical measures, crossvalidated correlation coefficient, external test set and randomization test, and through observation on how it reproduces and explains the quantitative differences seen in the experimentally known activity data. The models are considered predictive model as the validation methods provided significant statistical parameters with $\mathrm{q}^{2}=0.880,0.623$ and pred_r $\mathrm{r}^{2}=0.830,0.714$, respectively for model 1 and 2. The developed 2D QSAR models revealed the importance of SlogP, T_N_O_5, Mol.Wt and SsBrE-index properties of compounds in their antidiabetic activity. These results will be an essential guide for the further design and development of new lead compounds of more potent antidiabetic compounds.

These studies can be further extended to develop QSAR models using some other approaches HQSAR, PARM, 3D QSAR and docking analysis of direct drug designing and further validation of the results obtained in the present studies. The field is further open for designing, synthesis and biological evaluation of potent antidiabetic compounds, pharmacokinetic studies and clinical studies to establish those molecules as drug.

\section{Conflict of interest}

The authors confirm that this article content has no conflicts of interest.

\section{References}

[1]. J.L. Wolff, B. Starfield, G.F. Anderson, Prevalence, expenditures, and complications of multiple chronic conditions in the elderly, Arch Internal Medicine 162 (2002) 2269-2276.

[2]. K.M. Narayan, J.P. Boyle, L.S. Geiss, Impact of recent increase in incidence on future diabetes burden U.S., 2005-2050, Diabetes Care 29 (2006) 2114-2116.

[3]. R.A. Anderson, Chromium in the prevention of diabetes, Diabetes Metabolism 26 (2000) 22-27.

[4]. W.T. Cefalu, F.B. Hu, Role of chromium in human health and in diabetes, Diabetic Care 27 (2004) 2741-2751.

[5]. A. D. Vaibhav, V. B. Prasad, SAR and computer aided drug design approaches in the discovery of peroxisome proliferator activated receptor $\gamma$ activators: a perspective, Journal of Computational Medicine (2013) Article ID 406049.
[6]. R.B. Tripathi, J. Jain, A.W. Siddiqui, Design of new peroxisome proliferators gamma activated receptor agonists (PPAR $\gamma$ ) via QSAR based modeling, Journal of Applied Pharmaceutical Science and Research 1 (2018) 23-26.

[7]. S. Q. Pantaleao, D.G.V. Fujii, V.G. Maltarollo, D.C. Silva, G.H.G. Trossini, K.C. Weber, L.P.B. Scott, K.M. Honorio, The role of QSAR and virtual screening studies in Type 2 diabetes drug discovery, Medicinal Chemistry 13 (2017) 706-720.

[8]. A.M. Chawla, P.Y. Chawla, R.K. Dhawan, QSAR study of 2,4-dioxothiazolidine antidiabetic compounds, Der Pharmacia Chemica 6 (2014) 103110.

[9]. S. Kesar, P. Mishra, P. Ojha, S. Singh, 2D QSAR study of potent GSK3 $\beta$ inhibitor for treatment of type II diabetes, International Journal of Pharmaceutical Science and Research 7 (2016) 2932-2943.

[10]. J.V. Zivkovic, N.V. Trutic, J.B. Veselinovic, G.M. Nikolic, A.M. Veselinovic, Monte Carlo method based QSAR modelling of maleimide derivatives as glycogensynthasekinase-3 $\beta$ inhibitors, Computers in Biology and Medicine 64 (2015) 276-282.

[11]. V.K. Vyas, H.G. Bhatt, P.K. Patel, J. Jalu, C. Chintha, N. Gupta, M. Ghate, CoMFA and CoMSIA studies on C-aryl glucoside SGLT2 inhibitors as potential antidiabetic agents, SAR and QSAR in Environmental Research 24 (2013) 519551.

[12]. M. Lorca, C. Morales-Verdejo, D. VásquezVelásquez, J. Andrades-Lagos, J. CampaniniSalinas, J. Soto-Delgado, G. Recabarren-Gajardo, J. Mella, Structure activity relationships based on 3DQSAR CoMFA/CoMSIA and design of aryloxypropanol-amine agonists with selectivity for the human $\beta 3$-adrenergic receptor and anti-obesity and antidiabetic profiles, Molecules 23 (2018) 1191.

[13]. K.M. Manoj, K. Rajnish, M. Priyanka, In silico accounting of novel pyridazine analogues as h-PTP 1B inhibitors: pharmacophore modelling, atombased 3D QSAR and docking studies, Medicinal Chemistry Research 23 (2014) 2701-2711.

[14]. L.L. Chang, K.L. Sidler, M.A. Cascieri, S.D. Laszlo, G. Koch, B. Li, M. MacCoss, N. Mantlo, S. O'Keefe, M. Pang, A. Rolando, W.K. Hagmann, Substituted imidazoles as glucagon receptor antagonists, Bioorganic and Medicinal Chemistry Letters 11 (2001) 2549-2553.

[15]. J. Borras, A. Scozzafava, L. Menabuoni, F. Mincione, F. Briganti, G. Minicione, C.T. Supuran, Carbonic anhydrase inhibitors: synthesis of watersoluble, topically effective intraocular pressure lowering aromatic/heterocyclic sulfonamides containing 8-quinoline-sulfonyl moieties: is the tail more important than the ring?, Bioorganic and Medicinal Chemistry 7 (1999) 2397-2406.

[16]. D. Vullo, M. Franchi, E. Gallori, J. Antel, A. Scozzafava, C.T. Supuran, Carbonic anhydrase inhibitors. Inhibition of mitochondrial isozyme V with aromatic and heterocyclic sulfonamides, 
Journal of Medicinal Chemistry 47 (2004) 12721279.

[17]. J.Y. Winum, J.M. Dogne, A. Casini, X. De Leval, J.L. Montero, A. Scozzafava, D. Vullo, A. Innocenti, C.T. Supuran, Carbonic anhydrase inhibitors: Synthesis and inhibition of cytosolic membrane-associated carbonic anhydrase isozymes I, II, and IX with sulfonamides incorporating hydrazino moieties, Journal of Medicinal Chemistry 48 (2005) 2121-2125.

[18]. V. Ravichandran, K. Venkateskumar, S. Shalini, R. Harish, Exploring the structure activity relationship of oxazolidinones as HIV-1 protease inhibitorsQSAR and pharmacophore modelling studies, Chemometerics and Intelligent Laboratory Systems 154 (2016) 52-61.

[19]. V. Ravichandran, O.C. Chean, D. K. Subramaniam, N.M. Ying, S. Sivadasan, H. Rajak, A. Rasheed, Designing hypothesis of diaryl pyrimidine analogs as anti-HIV agent: QSAR approach, Medicinal Chemistry Research 22 (2013) 35-44.

[20]. A. Guyon, A. Elisseeff, An introduction to variable and feature selection, Journal of Machine Learning Research 3 (2003) 1157-1182.
[21]. R.B. Darlington, Regression and linear models, McGraw-Hill Higher Edu., New York, 23 (1990) 365-377.

[22]. K. Hasegawa, T. Kimura, K. Funatsu, GA strategy for variable selection in QSAR studies: enhancement of comparative molecular binding energy analysis by GA based PLS method, Quantitative Structure Activity Relationship 18 (1999) 262-272.

[23]. W. Zheng, A. Tropsha, Novel variable selection quantitative structure-property relationship approach based on the k-Nearest-Neighbor principle, Journal of Chemical Informatics and Computer Science 40 (2000) 185-194.

[24]. V. Ravichandran, H. Rajak, A.K. Jain, S. Shalini, P.V. Christapher, R.K. Agrawal, Validation of QSAR models - Strategies and importance, International Journal of Drug Design and Discovery 2 (2011) 511-519

Received: 23.12 .2018

Received in revised form: 13.02.2019

Accepted: 13.02.2019 